\title{
VIETA'S TRIANGULAR ARRAY AND A RELATED FAMILY OF POLYNOMIALS
}

\author{
NEVILLE ROBBINS \\ Department of Mathematics \\ San Francisco State University \\ San Francisco, CA 94132 \\ (Received November 2, 1988 and in revised form December 12, 1988)
}

\begin{abstract}
If $n \geq 1$, let the $n^{\text {th }}$ row of an infinite triangular array consist of entries $B(n, j)=\frac{n}{n-j}\left(\begin{array}{c}n-j \\ j\end{array}\right)$, where $0 \leq j \leq\left[\frac{1}{2} n\right]$.
\end{abstract}

We develop some properties of this array, which was discovered by Vieta. In addition, we prove some irreducibility properties of the family of polynomials $\left.V_{n}(x)=\sum_{j}^{\left[\frac{1}{2} n\right]}\right)(-1)^{j} B(n, j) x^{n-2 J}$.

These polynomials, which we call Vieta polynomials, are related to Chebychev polynomials of the first kind.

KEY WORDS AND PHRASES: Binomial coefficient, Fibonacci number, Lucas number, irreducible polynomial, Chebychev polynomial.

1980 AMS SUBJECT CLASSIFICATION CODE: 10A35, 10A40

\section{INTRODUCTION.}

For any $n \geq 1$, we let the $n^{\text {th }}$ row of an infinite left adjusted triangular array consist of the entries

$$
\begin{aligned}
B(n, j) & =\frac{n}{n-j}\left(\frac{n-j}{j}\right), 0 \leq j \leq\left[\frac{1}{2} n\right] \\
& = \begin{cases}1 & \text { if } j=0 \\
\frac{n}{j}\left({ }^{n}-j-1\right. & \text { if } 1 \leq j \leq\left[\frac{1}{2} n\right]\end{cases}
\end{aligned}
$$

where Horadam [1] attributes (1.1) to Lucas [2]. The array in question is

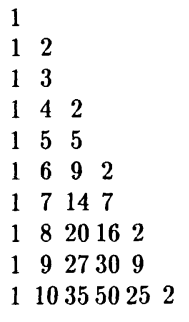


This array was first used by Vieta [3], where we find

$$
2 \cos n \theta=\sum_{j=0}^{\left[\frac{1}{2} n\right]}(-1)^{j} B(n, j)(2 \cos \theta)^{n-2 J}
$$

The array has also been studied by Lucas [2] who observed (1.2) as well as

$$
x^{n}+y^{n}=\sum_{J=0}^{\left[\frac{1}{2} n\right]}(-1)^{\jmath} B(n, j)(x y)^{\jmath}(x+y)^{n-2 \jmath}
$$

which is attributed to Lagrange by Ribenboim [4,p. 45],

and for odd $n$

$$
\begin{gathered}
L_{k n}=\sum_{j=0}^{\left[\frac{1}{2} n\right]}(-1)^{\jmath(k+1)} B(n, j) L_{k}^{n-2 \jmath} \\
F_{k n}=\sum_{j=0}^{\left[\frac{1}{2} n\right]}(-1)^{k j} 5^{\frac{1}{2}(n-1-2 \jmath)} F_{k}^{n-2 \jmath}
\end{gathered}
$$

where $L_{m}$ and $F_{m}$ are respectively the $m^{\text {th }}$ Lucas and Fibonacci numbers. Equations (1.5) and (1.6) readily follow by induction on $\mathrm{n}$. In Hoggatt [5], we find

$$
L_{n}=\sum_{j=0}^{\left[\frac{1}{2} n\right]} B(n, j)
$$

which follows directly from (1.5) with $\mathrm{k}=1$, and

$$
B(n, j)=B(n-1), j)+B(n-2, j-1), n \geq 3 \text { and } 0 \leq j \leq\left[\frac{1}{2} n\right]
$$

where it is assumed that a zero is used whenever a number in the array does not appear.

Equations $(1.4),(1.7)$ and $(1.8)$ illustrate that the $[B(n, j)]$ have properties similar to those of the binomial coefficients.

In this paper, we develop additional properties associated with this array as well as prove some irreducibility properties of the family of polynomials

$$
V_{n}(x)=\sum_{j=0}^{\left[\frac{1}{\left.2^{n}\right]}\right.}(-1)^{j} B(n, j) x^{n-2 \jmath}
$$

which we call Vieta polynomials.

Jacobstahl [6] proved that $\left\{V_{n}(x)\right\}$ is a family of permutable polynomials, that is, $V_{m}\left(V_{n}(x)\right)=V_{n}\left(V_{m}(x)\right)=V_{m n}(x)$ for all natural numbers $m, n$. He also proved that if $\left\{f_{n}(x)\right\}$ is a family of permutable polynomials such that the degree of $f_{n}(x)$ is $\mathrm{n}$ for all $\mathrm{n}$, then $f_{n}(x)$ is similar to either $V_{n}(x)$ or $x^{n}$, that is, there exists a polynomial $g(x)=a x+b$ such that $f_{n}(x)=g(x){ }^{*} V_{n}(x){ }^{*} g^{-1}(x)$ or $f_{n}(x)=g(x){ }^{*} x^{n}{ }^{*} g^{-1}(x)$, where * denotes composition. Jacobstahl also noted that the Vieta polynomials are related to the Chebychev polynomials of the first kind (denoted $T_{n}(x)$ ) by the identity:

$$
V_{n}(x)=2 T_{n}\left(\frac{1}{2} x\right)
$$

In addition, he gave an inductive definition for the $V_{n}(x)$, namely:

$$
V_{0}(x)=2, V_{1}(x)=x, V_{n}(x)=x V_{n-1}(x)-V_{n-2}(x), n \geq 2
$$


The Lucas polynomials, defined by Bicknell in [7] as

$$
L_{0}(x)=2, L_{1}(x)=x, L_{n}(x)=x L_{n-1}(x)+L_{n-2}(x), n \geq 2
$$

are not permutable and satisfy

$$
L_{n}(x)=\sum_{j=0}^{\left[\frac{1}{2} n\right]} B(n, j) x^{n-2 j}
$$

as well as

$$
V_{n}(i x)=i^{n} L_{n}(x)
$$

where $i=(-1)^{\frac{1}{2}}$

In conclusion, we point out that Bergum and Hoggatt [8] prove that if $k \geq 1$ and $p$ is an odd prime, then $L_{2} k(x)$ and $L_{p}(x) / x$ are irreducible over the rationals. Their proofs adapt easily to show that the same properties hold respectively for $V_{2} k(x)$ and $V_{p}(x) / x$. In this paper, we generalize the latter result. Specifically, we prove that if $m \geq 1, \mathrm{p}$ and $\mathrm{n}$ are odd, and $p$ is prime, then both $V_{p n}(x) / V_{n}(x)$ and $V_{3\left(2^{m}\right)}(x) / V_{2^{m}}(x)$ are irreducible over the rationals.

2. PRELIMINARIES.

Below is a list of identities, some of which are used to develop the results in section 3 of this paper, while others are of interest in their own right.

Using (1.9) and (1.10) in Rivlin [9], with (1.10), we have

$$
B(n, j)=\frac{1}{2^{n-1-2 j}} \sum_{k=j}^{\left[\frac{1}{2} n\right]}\left(\begin{array}{l}
n \\
2 k
\end{array}\right)(k)
$$

Letting $x=y^{-1}=e^{\theta}$ in (1.4), we have

$$
2 \cosh n \theta=\sum_{j=0}^{\left[\frac{1}{2} n\right]}(-1)^{\jmath} B(n, j)(2 \cosh \theta)^{n-2 J}
$$

while

$$
o_{p}(B(n, j)) \geq o_{p}(n)-o_{p}(j)
$$

where $o_{p}(n)=h$ if $p^{h} \mid n, p^{h+1} \gamma_{n, h} \geq 0$, follows directly from (1.2) and the laws of exponents.

In Cohn [10, p. 156], we find

$$
\begin{aligned}
& x^{2}-5 y^{2}=-4 \text { if there exists an odd } k \text { such that } \\
& x=L_{k}, y=F_{k}
\end{aligned}
$$

while

$$
V_{m n}(x)=V_{m}\left(V_{n}(x)\right) \text { for all } m, n
$$

appears in [6].

Letting $x=y^{-1}=e^{s \theta}$ in (1.4), we have

$$
2 \cos n \theta=\sum_{j=0}^{\left[\frac{1}{2} n\right]}(-1)^{j} B(n, j)(2 \cos \theta)^{n-2 \jmath}
$$


Using (1.9) with (2.6) and (2.2), we obtain

$$
V_{n}(2 \cos \theta)=2 \cos n \theta
$$

and

$$
V_{n}(2 \cosh \theta)=2 \cosh n \theta
$$

Finally, we observe that it is easy to show that

$$
5 F_{4 m+1}-L_{4 m+1} \equiv \pm 4(\bmod 10), m \geq 0
$$

\section{THE MAIN RESULTS.}

We begin with some divisibility properties of the $B(n, j)$.

THEOREM 1. If $1 \leq j \leq\left[\frac{n-1}{2}\right], p^{h} \mid n$, and $p \nmid j$, then $p^{h} \mid B(n, j)$.

PROOF. This follows from the hypothesis and (2.3).

THEOREM 2. If $1 \leq j \leq\left[\frac{1}{2} n\right]$ and $(n, j)=1$, then $n \mid B(n, j)$.

PROOF. Equation (1.2) implies $n \mid j B(n, j)$. The conclusion now follows from the hypothesis and Euclid's Lemma.

COROLLARY 1. If $\mathrm{p}$ is an odd prime and $1 \leq j \leq \frac{p-1}{2}$, then $p \mid B(p, j)$.

PROOF. This follows directly from Theorem 2 .

THEOREM 3. For all $j$ such that $1 \leq j \leq\left[\frac{n-1}{2}\right]$ there exists a prime, $p$, such that $p \mid n$ and $p \mid B(n, j)$.

PROOF. Assume the contrary. Then there exists $j$ such that $1 \leq j \leq\left[\frac{n-1}{2}\right]$ and for all primes $p$ where $p \mid n$ we have $p \nmid B(n, j)$, so $o_{p}(B(n, j))=0$. But then (2.3) implies $o_{p}(n) \leq o_{p}(j)$. Since this inequality holds for all prime divisors of $n$, we have $n \mid j$, which is contrary to our hypothesis.

COROLLARY 2. If $1 \leq j \leq\left[\frac{1}{2} p^{k}\right]$, then $p \mid B\left(p^{k}, j\right)$.

PROOF. This follows from Theorem 3.

THEOREM 4. Let $\mathrm{p}$ be an odd prime. If $1 \leq j \leq p^{k}$, then $p \mid B\left(2 p^{k}, j\right)$. If $1 \leq j \leq 2 p^{k}$ and $j \neq p^{k}$, then $p \mid B\left(4 p^{k}, j\right)$.

PROOF. The hypothesis implies that $o_{p}(j) \leq k-1$, so the conclusions follow from (2.3).

THEOREM 5. If $o_{p}(n)=1$, then $p / B(n, p)$.

PROOF. The hypothesis implies that $n=k p$ and $p / k$. Since (1.2) implies that $B(n, p)=B(k p, p)=k \frac{p-1}{11} \frac{k p-p-i}{p-i}$, the conclusion follows directly from the fact that $p \nmid \frac{p-1}{i 1}(k p-p-i)$.

The next result provides an analog to the identity $\sum_{j=0}^{n}(-1)^{\jmath}\left(\begin{array}{l}n \\ j\end{array}\right)=0$.

THEOREM 6. $\sum_{J=0}^{\left[\frac{1}{2} n\right]}(-1)^{j} B(n, j)=\left\{\begin{array}{c}2 \text { if } n \equiv 0(\bmod 6), 1 \text { if } n \equiv \pm 1(\bmod 6), \\ -1 \text { if } n \equiv \pm 2(\bmod 6),-2 \text { if } n \equiv 3(\bmod 6)\end{array}\right.$

PROOF. This follows from (1.3) with $\theta=\pi / 3$.

The next theorem locates the largest entry or entries in the $n^{\text {th }}$ row of Vieta's triangular array.

THEOREM 7. If $0 \leq j \leq\left[\frac{1}{2} n\right]$, let $r=\left(5 n+6-\left(5 n^{2}-4\right)^{\frac{1}{2}}\right) / 10$.

If $\mathrm{r}$ is not an integer, then $B(n,[r])>B(n, j)$ for all $j \neq[r]$.

If $\mathrm{r}$ is an integer, then $B(n, r-1)=B(n, r)>B(n, j)$ for all $j \neq r-1, r$. 
PROOF. Let $f_{n}(j)=\frac{B(n, j)}{B(n, j-1)}$ where $1 \leq j \leq\left[\frac{1}{2} n\right]$. Equation (1.2) implies that $f_{n}(j)=\frac{n(n-j-1) !}{j !(n-2 j !} * \frac{(j-1) !(n-2 j+2) !}{n(n-j) !}=\frac{(n+1-2 j)(n+2-2 \jmath)}{j(n-j)}$.

Now $B(n, j)$, considered as a function of $j$ for a fixed value of $n$, is increasing, decreasing, or not changing accordingly as $f_{n}(j)$ is greater than, less than, or equal to 1 . These possibilities occur accordingly as $5 j^{2}-(5 n+6) j+(n+1)(n+2)$ is positive, negative, or zero, or accordingly as $1 \leq j<r, j=r$, or $r<j \leq\left[\frac{1}{2} n\right]$.

If $\mathrm{r}$ is not an integer, then $B(n, j)$ is increasing for $1 \leq j \leq[r]$ and decreasing for $[r] \leq j \leq\left[\frac{1}{2} n\right]$. Hence, $B(n, j)$ assumes a unique maximum at $j=[r]$. If $\mathrm{r}$ is an integer, then $B(n, r-1)=B(n, r)$ and $B(n, j)$ is increasing for $1 \leq j \leq r-1$, decreasing for $r \leq j \leq\left[\frac{1}{2} n\right]$. Hence, $B(n, j)$ assumes its maximum value when $j=r-1$ or $r$.

The following two theorems give necessary and sufficient conditions for $r$ to be an integer.

THEOREM 8. $\left(5 F_{4 m+1}-L_{4 m+1}+6\right) / 10=F_{2 m}^{2}+1$.

PROOF. This follows directly by induction, or by using Binet's formulas for $F_{n}$ and $L_{n}$.

THEOREM 9. Let $r=\left(5 n+6-\left(5 n^{2}-4\right)^{\frac{1}{2}} / 10\right.$. Then $\mathrm{r}$ is an integer if there exists $m$ such that $n=F_{4 m+1}$ and $r=F_{2 m}^{2}+1$.

PROOF. If $\mathrm{r}$ is an integer, then $\left(5 n^{2}-4\right)^{\frac{1}{2}}=t$ is an integer.

Therefore, $t^{2}-5 n^{2}=-4$ and (2.4) implies that there exists an odd $\mathrm{k}$ such that $t=L_{k}, n=F_{k}$. Since $\mathrm{r}$ is an integer, we must also have $5 F_{k}-L_{k} \equiv 4(\bmod 10)$. Since $(2.9)$ implies that $k=4 m+1$, we have $r=\left(5 F_{4 m+1}-L_{4 m+1}+6\right) / 10$. Theorem 8 now implies that $r=F_{2 m}^{2}+1$. The converse clearly holds.

We now turn our attention to the Vieta polynomials.

THEOREM 10. If $\mathrm{n}$ is odd and $\mathrm{p}$ is an odd prime, then $V_{p n}(x) / V_{n}(x)$ is irreducible over the rational numbers.

PROOF. Using (2.5) and (1.9), we see that

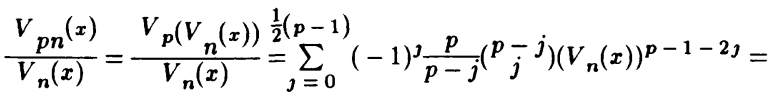

$$
\begin{aligned}
& \sum_{j=0}^{\frac{1}{2}(p-1)}(-1)^{j} \frac{p}{p-j}\left({ }^{p-j} j^{\frac{1}{2}(n-1)}\left(\sum_{i=0}^{n}(-1)^{i} \frac{n}{n-i}\left({ }^{n} i^{\imath}\right) x^{n-2 i}\right)^{p-1-2 j .}\right.
\end{aligned}
$$

The lead term is $x^{(p-1) n}$ while all other variable terms are divisible by $\mathrm{p}$. The constant term is $p(-1)^{\frac{1}{2}(p-1)}$. Therefore, the conclusion follows from the Eisenstein criterion.

One may conjecture that the conclusion of Theorem 10 is also valid when $\mathrm{n}$ is even, but this difficult to prove.

LEMMA 1. $V_{2 m p}(0) / V_{2 m}(0)=1$ if $p$ is odd.

PROOF. Equation (2.6) implies that $V_{2 m}(0)=V_{2 m}\left(2 \cos \frac{1}{2} \pi\right)=2 \cos m \pi=2(-1)^{m}$. Therefore, $V_{2 m p}(0) / V_{2 m}(0)=2(-1)^{p m} / 2(-1)^{m}=(-1)^{(p-1) m}=1$.

REMARK. Lemma 1 says that constant term in $V_{p n}(x) / V_{n}(x)$ is 1 if $\mathrm{n}$ is even, so that Eisenstein criterion is not immediately applicable to prove irreducibility.

THEOREM 11. Let $g_{m}(x)=V_{2^{m}}(x)-1$, where $m \geq 0$. Then $g_{m}(x)$ is irreducible over the rational numbers.

PROOF. (Induction on $\mathrm{m}$ ) For $m=0, g_{0}(x)=V_{1}(x)-1=x-1$, which is irreducible. If $m \geq 1$, let $h_{m}(y)=g_{m}(y+1)=V_{2^{m}}(y+1)-1$. Then

$$
h_{m}(0)=g_{m}(1)=V_{2^{m}}(1)-1=V_{2^{m}}\left(2 \cos \frac{\pi}{3}\right)-1=2 \cos \left(2^{m} \pi / 3\right)-1=2\left(-\frac{1}{2}\right)-1=-2 .
$$


But

$$
h_{1}(y)=V_{2}(y+1)-1=(y+1)^{2}-2-1=y^{2}+2 y-2,
$$

and

$$
h_{m}(y)=2_{j=0}^{m}-1(-1)^{\jmath} B\left(2^{m}, j\right)(y+1)^{2^{m}-2 \jmath}-1=y^{2^{m}}+\sum_{k=1}^{2^{m}-1} c_{k, m} y^{2^{m}-k}-2 .
$$

We claim that each coefficient $c_{k, m}$ is even. To prove this, we note that

$$
\begin{gathered}
h_{m+1}(y)=V_{2^{m+1}}(y+1)-1=V_{2}\left(V_{2^{m}}(y+1)\right)-1=V_{2}\left(h_{m}(y)+1\right)-1=\left(h_{m}(y)+1\right)^{2}-3= \\
\left(y^{2^{m}}+\sum_{k=1}^{2^{m}-1} c_{k, m} y^{2^{m}-k}-1\right)^{2}-3=y^{2^{m+1}}+\sum_{k=1}^{m+1} c_{k, m+1} y^{2^{m+1}}-k-2 .
\end{gathered}
$$

Since (by induction hypothesis) all the $c_{k, m}$ are even, it follows that all the $c_{k, m+1}$ are even. Therefore, $h_{m}(y)$ is irreducible over the rational numbers by the Eisenstein criterion, with $\mathrm{p}=2$. Letting $\mathrm{x}=\mathrm{y}+1$, we have $g_{m}(x)$ is irreducible over the rationals for all $m \geq 0$. rationals.

THEOREM 12. If $f_{m}(x)=V_{3\left(2^{m}\right)}(x) / V_{2^{m}}(x)$ where $m \geq 0$, then $f_{m}(x)$ is irreducible over the

PROOF. $f_{m}(x)=\left(V_{2^{m}}(x)\right)^{2}-3=V_{2^{m+1}}(x)-1=g_{m+1}(x)$, so the conclusion follows from Theorem 11.

\section{REFERENCES}

1. A.F. HORADAM "Tschebyscheff and Other Functions Associated with the Sequence Fibonacci Quart. 7 (1969) 14-22

2. E. LUCAS "Theorie des functions Numeriques Simplement Periodiques" Amer. J. Math. 1 (1878) 184-240; 289-321.

3. F. VIETA "Opera Mathematica: Ad Angulas Sectiones" (Theorema VI) Paris, 1615.

4. P. RIBENBOIM "The Book of Prime Number Records" Springer-Verlag 1988.

5. V.E. HOGGATT "An Application of the Lucas Triangle" Fibonacci Quart. 8 (1970) 360-364.

6. E. JACOBSTAHL "Ueber Vertauschbare Polynome" Math. Zeits. $\underline{63}$ (1955) 244-276.

7. M. BICKNELL "A Primer for the Fibonacci Numbers: Part VII" Fibonacci Quart. 8 (1970) 407-420.

8. G.E. BERGUM \& V.E. HOGGATT "Irreducibility of Lucas and Generalized Lucas Polynomials" Fibonacci Quart. 12 (1974) 95-100.

9. T. RIVLIN "The Chebyshev Polynomials" Wiley-Interscience 1974.

10. J.H.E. COHN "Eight Diophantine Equations" Proc. London Math Soc. (3) 16 (1966) 153-166. 


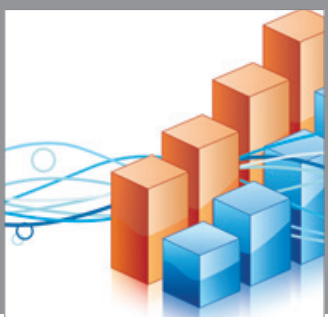

Advances in

Operations Research

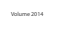

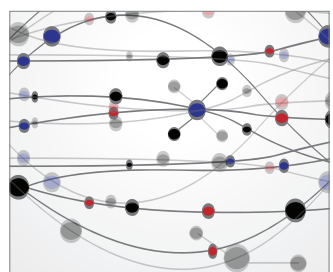

\section{The Scientific} World Journal
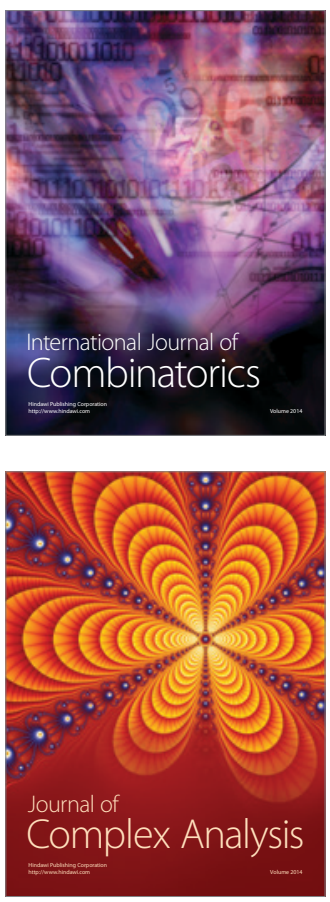

International Journal of

Mathematics and

Mathematical

Sciences
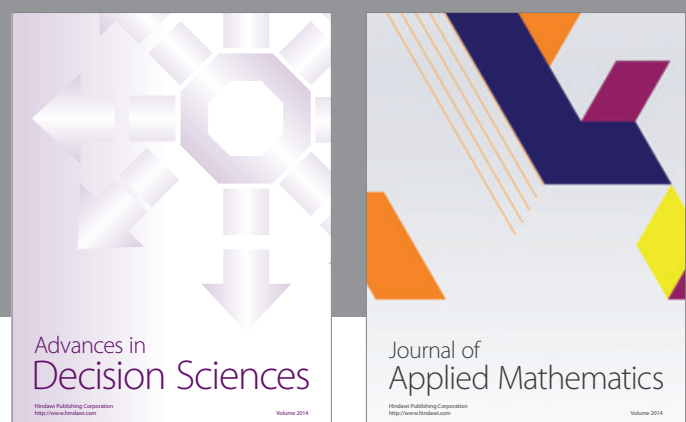

Journal of

Applied Mathematics
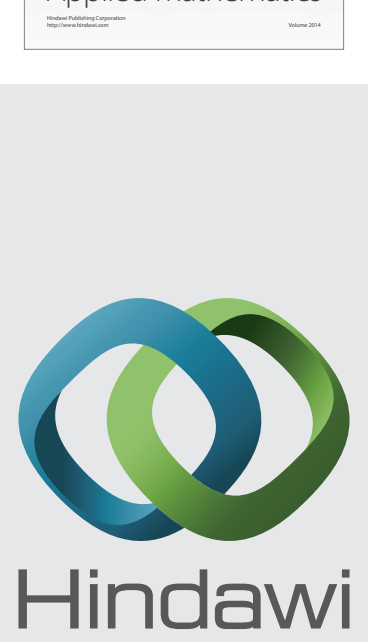

Submit your manuscripts at http://www.hindawi.com
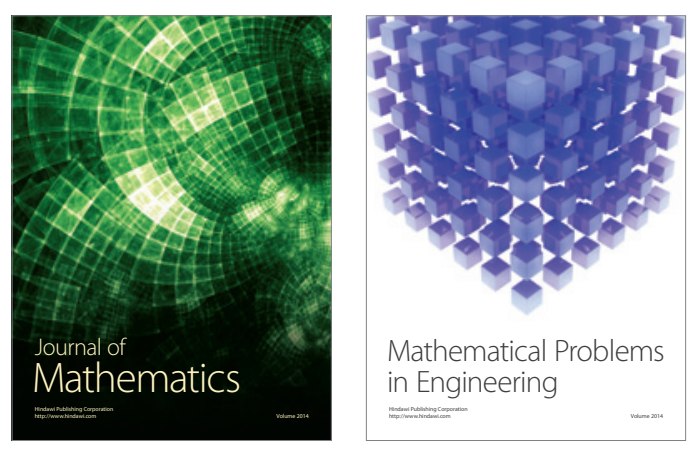

Mathematical Problems in Engineering
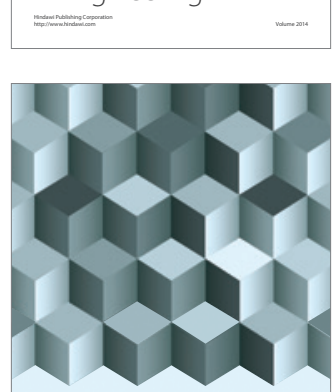

Journal of

Function Spaces
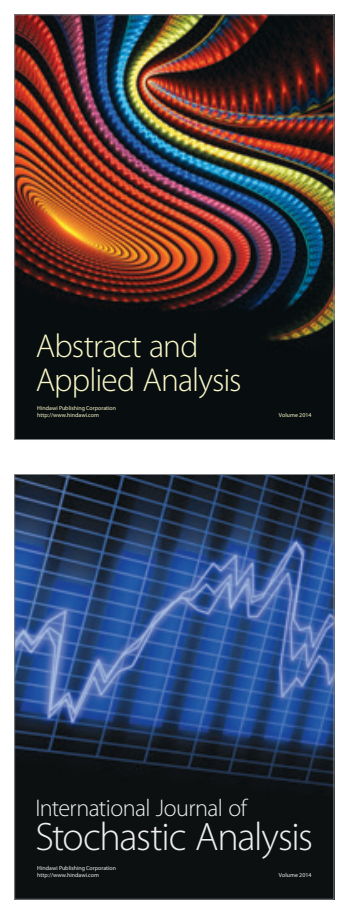

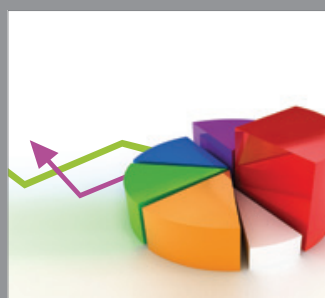

ournal of

Probability and Statistics

Promensencen
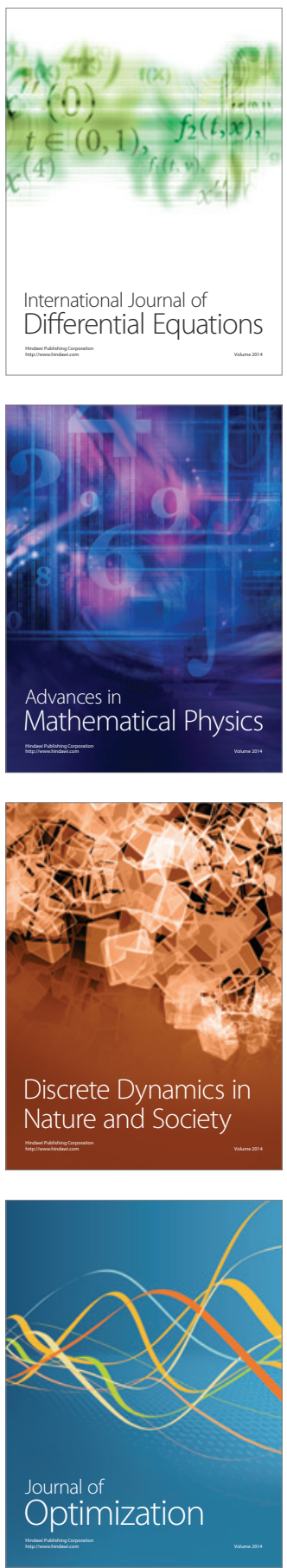